\title{
Clinical study on cluster care to prevent multi-drug resistant infection in ICU patients with severe encephalopathy
}

\author{
LIU QIONG and ZHU HUI \\ Jiangyin People's Hospital of Brain ICU, Jiangyin, Jiangsu 214400, P.R. China \\ Received January 12, 2016; Accepted May 16, 2016
}

DOI: $10.3892 /$ etm.2016.3876

\begin{abstract}
The value of clinical cluster nursing in the prevention of multi-drug resistant (MDR) infection in patients with severe encephalopathy in ICU was evaluated. ICU patients $(n=129)$ diagnosed with severe encephalopathy between 2012 and 2014 were selected as the study group, while 106 cases of ICU patients diagnosed with severe encephalopathy between 2010 and 2012 were retrospectively selected as the control group. Control group patients were offered conventional integrated nursing care, while the study group patients were offered cluster nursing care. The differences in infection rate, colony and quantity, infection time, number, mortality rate and hospital stays between the two groups were compared and analyzed. Observations on the infection rate, diagnosis time, total number of infection, mortality rate caused by infection and hospital stays were lower in the study group patients than in controls $(\mathrm{P}<0.05)$. The patients in the study group had a much lower drug-resistant infection rate than that in the control group $(\mathrm{P}<0.05)$. In the patient groups there were infections with methicillin-resistant Staphylococcus aureus, Pseudomonas aeruginosa, Klebsiella pneumoniae and Escherichia coli, although the quantities of the above pathogenic microbe colonies in the study group were notably less than those in the control group $(\mathrm{P}<0.05)$. In conclusion, cluster nursing care effectively prevents MDR infections of ICU patients with severe encephalopathy and reduces the mortality rate, thus having an excellent clinical significance.
\end{abstract}

\section{Introduction}

Severe encephalopathy patients exhibit acute disease onset, focal or diffuse brain function loss, including acute cerebral hemorrhage, cerebral infarction, subarachnoid hemorrhage, severe encephalitis and other diseases (1). Severe

Correspondence to: Dr Liu Qiong, Jiangyin People's Hospital of Brain ICU, 163 Shoushan Road, Jiangyin, Jiangsu 214400, P.R. China

E-mail: mpuqruhqtp322@163.com

Key words: cluster nursing care, severe encephalopathy, multiple drug-resistant organism, infection, mortality rate encephalopathy is a set clinical syndrome with high incidence, high disability rate, and high mortality rate (1).

The critical condition of patients, long course, poor resistance to infection, coma, dysphagia, many invasive procedures including tracheal intubation or incision, a large number of pathogens and drug-resistant bacteria in ICU and the improper preventive use of antibiotics, contribute to cross-infection and multi-drug resistant (MDRs) infections (2). Cluster nursing care is the application of evidence-based medicine, bundling a series of independent and effective operations, care, and treatment measures, in order to reduce the infection rate in patients and to improve the clinical remedy rate (3). Overall, cluster nursing care can achieve significantly better results than the intervention of single factor care.

The present study investigated the applied value of cluster nursing interventions in the prevention of MDR bacterial infection in ICU patients with severe encephalopathy.

\section{Patients and methods}

Patients. The present study was approved by the Ethics Committee of the Jiangyin People's Hospital of Brain ICU and consent was obtained from the patients or their families. ICU patients $(n=129)$ diagnosed with severe encephalopathy between August 2012 and August 2014 were continuously selected as the study group, with concurrently infected patients or potentially infected patients being excluded. In addition, 106 cases of ICU patients diagnosed with severe encephalopathy between August 2010 and August 2012 were retrospectively selected as the control group.

The study group comprised 76 male and 53 female cases, with an age range of 43-82 years and an average age of $58.6 \pm 10.2$ years. The disease course was from 1 h to 18 days with an average course of $6.7 \pm 1.3$ days. There were 96 cases of consciousness disturbance, 103 cases of respirator usage (84 cases of tracheal cannula, and 19 cases of tracheotomy), 78 cases of central vein catheter, and 115 cases of retention catheterization. The control group comprised 64 men and 42 women, with an age range of 38-78 years. and an average age of $57.5 \pm 11.4$ years. The disease course was from $5 \mathrm{~h}$ to 29 days with an average of $6.4 \pm 1.5$ days. There were 84 cases of consciousness disturbance, 95 cases of respirator usage (74 cases of tracheal cannula, 21 cases of tracheotomy), 98 cases of central vein catheter, and 100 cases of retention catheterization. There were no statistically significant 
differences between the two groups concerning gender, age, course of disease, consciousness disturbance, usage of respirator, central vein catheter and retention catheterization.

Cluster care approach. The control group was under standard conventional integrated nursing care, which was as follows: one nurse was in charge of 2-3 beds, sterilizing the patients, respirators and disinfection operations three times a day, once in the morning, once at noon and another in the evening, and made a regular bacterial inspection report.

The study group was under cluster nursing intervention model, which was as follows (4,5): i) A maintenance team dealing with infection prevention was established, with the team members undergoing professional training. A specifically assigned individual was responsible for maintenance and was assessed on a monthly basis. ii) Standardized preventive measures for patients were implemented. Suspected patients with multiple drug-resistant infections, as well as the closed suction tube (Kangnuo,Suzhou, China), which was in touch with the infected patients were quarantined; iii) maintenance of strict skin disinfection. The skin was stringently sterilized with chlorhexidine prior to intubation and the largest protection barrier was implemented; iv) use the anti-bacterial catheters. The catheter interface was wiped with an ethanol cotton piece and the catheter was fixed with $2 \%$ chlorhexidine patch. One maintenance was required to be carried out within $24 \mathrm{~h}$ after the day of puncture and sterilized dressing used to absorb cataclysm at the puncture point was replaced within $24 \mathrm{~h}$; v) attention was paid to hand hygiene and occupational protection. Each ward was required to be equipped with hand washing and hand-drying facilities. Each bed was equipped with quick-drying hand disinfectant. Medical personnel were required to wash hands according to the hand-washing guidelines. Although no direct infections are transmitted while wearing gloves, a thorough disinfection with quick-drying hand disinfectant was required; vi) the monitoring of patient multi-drug infectious organism was strengthened, and patients with potential MDR infectious organism were monitored and patients treated with broad-spectrum antimicrobials from other departments were admitted. Following admission, the drug sensitivity test was implemented continuously for three days to detect MDR infectious organism and carry out corresponding control and treatment measures according to the result; and vii) monitoring temperature, procalcitonin, hemogram, CRP and other infection indicators. If there are
Table I. Comparison of MDR infection rate between the two patient groups.

\begin{tabular}{lcccc}
\hline & $\begin{array}{c}\text { Control } \\
\text { group }(\mathrm{n}=106) \\
\mathrm{n}(\%)\end{array}$ & $\begin{array}{c}\text { Observation } \\
\text { group }(\mathrm{n}=129) \\
\mathrm{n}(\%)\end{array}$ & $\mathrm{t}\left(\chi^{2}\right)$ & P-value \\
Groups & $32(30.19)$ & $11(8.53)$ & 5.426 & $<0.001$ \\
\hline Infection rate & $7.52 \pm 0.68$ & $13.54 \pm 2.34$ & 4.521 & $<0.001$ \\
$\begin{array}{l}\text { Infection } \\
\text { diagnosis time }\end{array}$ & & & & \\
Infection times & $2.34 \pm 0.27$ & $1.29 \pm 0.31$ & 3.624 & 0.037 \\
$\begin{array}{l}\text { Infection } \\
\text { mortality rate }\end{array}$ & $21(19.81)$ & $7(5.43)$ & 4.798 & $<0.001$ \\
Hospital stays & $24.67 \pm 5.49$ & $16.45 \pm 5.23$ & 2.657 & 0.036 \\
\hline
\end{tabular}

MDR, multi-drug resistant.

any temperature anomalies, catheter infection was considered first. Blood samples were for culture, extracting peripheral blood and intraductal blood were extracted for aerobic and anaerobic cultures, respectively, and catheter tip was collected for culture. If catheter infection was identified, it was treated according to indications and reported to Department of Infection of the hospital.

Observed indicators. The differences of the infection rate, infectious organism colonies and the quantity, time, number, mortality rate and hospital stays between the two patient groups were compared and analyzed.

Statistical analysis. Data were analyzed using SPSS 19.0 statistical software (Chicago, IL, USA). Data are shown as mean \pm SD. The Student's t-test was employed for group comparisons. Case number or $(\%)$ were used to express qualitative data and the $\chi^{2}$ test was employed for inter-group comparisons. Differences were considered statistically significant at $\mathrm{P}<0.05$.

\section{Results}

MDR infection rate comparisons between two patient groups. The study group had a significantly lower $(\mathrm{P}<0.05)$ patient infection rate, infection diagnosis time, total number of infections, mortality rate caused by infections and hospital stays than the control group (Table I).

Table II. Comparisons on the infection colony number between the two patient groups.

\begin{tabular}{|c|c|c|c|c|}
\hline Groups & $\begin{array}{l}\text { Control group } \\
(n=106), n(\%)\end{array}$ & $\begin{array}{l}\text { Observation group } \\
\quad(\mathrm{n}=129), \mathrm{n}(\%)\end{array}$ & $\mathrm{t}\left(\chi^{2}\right)$ & P-value \\
\hline 1 type of drug-resistant bacteria & $5(4.72)$ & $4(3.10)$ & 2.356 & 0.028 \\
\hline 2 types & $16(15.09)$ & $5(3.88)$ & 5.324 & $<0.001$ \\
\hline 3 types or more & $11(10.38)$ & $2(1.55)$ & 6.529 & $<0.001$ \\
\hline Methicillin-resistant Staphylococcus aureus (CFU/ml) & $57.63 \pm 6.59$ & $32.04 \pm 8.47$ & 4.256 & $<0.001$ \\
\hline Pseudomonas aeruginosa $(\mathrm{CFU} / \mathrm{ml})$ & $35.64 \pm 4.27$ & $16.52 \pm 5.54$ & 5.127 & $<0.001$ \\
\hline Klebsiella pneumoniae (CFU/ml) & $24.18 \pm 5.13$ & $8.49 \pm 1.23$ & 6.128 & $<0.001$ \\
\hline Escherichia coli $(\mathrm{CFU} / \mathrm{ml})$ & $16.25 \pm 4.87$ & $5.17 \pm 1.14$ & 5.689 & $<0.001$ \\
\hline
\end{tabular}


Comparison of the infectious organism colonies and quantity between the two patient groups. Rates of 1,2,3 or more types of drug-resistant bacterial infection in patients in the study group were markedly lower than those in the control group and the differences were statically significant $(\mathrm{P}<0.05)$. The control group had a higher proportion of drug-resistant infections with 2,3 or more types, while the observation group had a higher proportion of drug-resistant infection with 1 and 2 types. The two patient groups were also had methicillin-resistant Staphylococcus aureus, Pseudomonas aeruginosa, Klebsiella pneumoniae and Escherichia coli colonies, although the quantity of the above colonies in the study group was significantly less than that of the control group $(\mathrm{P}<0.05)$ (Table II).

\section{Discussion}

The incidence of MDR infections of ICU patients with severe encephalopathy is becoming worse, making treatment thereof even more difficult. Previous findings have shown that cluster nursing care has notable effects on reducing the incidence rate of MDR infections in ICU patients (6). The priority for the prevention and treatment of MDR infections is to control their outbreak and spread, rather than passive treatment, and to carry out medical care on MDR-infected patients by trained medical professionals (7). Active monitoring of drug-resistant bacteria can be useful to detect, quarantine, diagnose earlier and treat the affected infected patients, earlier, thereby effectively preventing and controlling the outbreak and populace of MDR infections in hospital (8).

The present results suggest that the patients in the study group, who received cluster nursing care, were affected less by the MDR infections as compared to the controls, who received standard nursing care. By summarizing practical experience, the present study provides many practical and feasible measures, such as hand hygiene and compliance checks, and training of catheter maintenance personnel. Strengthening hand hygiene is the most economical and effective means to prevent and control hospital infection $(9,10)$. In the training of hand hygiene, hand hygiene awareness of the nursing staff should be improved, and correct ways of conducting hand hygiene, which involve aspects such as rubbing time, rubbing method and hand-drying method, should be mastered. Additionally, disinfection and quarantine system should be implemented (11). Quarantine measures may be carried out effectively on suspected MDR-infected patients. Ensuring that the medical supplies that came in contact with a patient are to be used just for that one patient and disinfection should be carried out effectively for those supplies that have to be used for more than one patient. Disposable devices must be utilized for oxygen inhalation by patients. Aseptic techniques and operation specifications must be implemented strictly and a variety of invasive operation indications must be mastered (3). In patients with deep venous catheters, the wound should be disinfected with $2 \%$ chlorhexidine solution and the puncture point should be protected with $10 \times 10 \mathrm{~cm}$ of $3 \mathrm{M}$ transparent dressing. Steps should be taken to promote and educate hospital infection knowledge with publication of relevant information documents and education of the patients and their families, and improve infection prevention awareness and strict prevention on cross-infection (12). Steps should be taken to actively prevent the infection by hypostatic pneumonia, and for proper oral care, and skin care, to prevent ventilator-related pneumonia, catheter-related urinary system infection and catheter-related blood infection and practice the preventive measures with cluster strategies (13).

In conclusion, the cluster nursing care can effectively prevent MDR infections of ICU patients with severe encephalopathy and reduce mortality rate, thus having a good application value in the clinic.

\section{References}

1. Young GB: Encephalopathy of infection and systemic inflammation. J Clin Neurophysiol 30: 454-461, 2013.

2. Tjia J, Mazor KM, Field T, Doherty P, Spenard A and Gurwitz JH: Predicting nursing home adherence to a clinical trial intervention: lessons for the conduct of cluster randomized trials. J Am Geriatr Soc 59: 2332-2336, 2011.

3. Sackley CM, van den Berg ME, Lett K, Patel S, Hollands K, Wright CC and Hoppitt TJ: Effects of a physiotherapy and occupational therapy intervention on mobility and activity in care home residents: a cluster randomised controlled trial. BMJ 339: b3123, 2009.

4. Shi L, Zhang J, Wang Y, Caulfield LE and Guyer B: Effectiveness of an educational intervention on complementary feeding practices and growth in rural China: a cluster randomised controlled trial. Public Health Nutr 13: 556-565, 2010.

5. Rico-Blázquez M, Escortell-Mayor E, Del-Cura-González I, SanzCuestaT, Gallego-Berciano P, de Las Casas-Cámara G, Soto-Díaz S, García-Sanz P, Harris-de-la-Vega N, Martín-Martín M, et al: CuidaCare: effectiveness of a nursing intervention on the quality of life's caregiver: cluster-randomized clinical trial. BMC Nurs 13: $2,2014$.

6. Jemmott JB III, Jemmott LS, O'Leary A, Ngwane Z, Icard LD, Heeren GA, Mtose X and Carty C: Cluster-randomized controlled trial of an HIV/sexually transmitted infection risk-reduction intervention for South African men. Am J Public Health 104: 467-473, 2014.

7. Oken E, Patel R, Guthrie LB, Vilchuck K, Bogdanovich N, Sergeichick N, Palmer TM, Kramer MS and Martin RM: Effects of an intervention to promote breastfeeding on maternal adiposity and blood pressure at $11.5 \mathrm{y}$ postpartum: results from the Promotion of Breastfeeding Intervention Trial, a cluster-randomized controlled trial. Am J Clin Nutr 98: 1048-1056, 2013.

8. Uchiyama A, Odagiri Y, Ohya Y, Takamiya T, Inoue $S$ and Shimomitsu T: Effect on mental health of a participatory intervention to improve psychosocial work environment: a cluster randomized controlled trial among nurses. J Occup Health 55: 173-183, 2013.

9. Hopkinson JB, Fenlon DR, Okamoto I, Wright DN, Scott I, Addington-Hall JM and Foster C: The deliverability, acceptability, and perceived effect of the Macmillan approach to weight loss and eating difficulties: a phase II, cluster-randomized, exploratory trial of a psychosocial intervention for weight- and eating-related distress in people with advanced cancer. J Pain Symptom Manage 40: 684-695, 2010

10. Jahn P, Kitzmantel M, Renz P, Kukk E, Kuss O, Thoke-Colberg A, Horn I and Landenberger M: Improvement of pain related self management for oncologic patients through a trans institutional modular nursing intervention: protocol of a cluster randomized multicenter trial. Trials 11: 29, 2010.

11. McNamara R, Robling M, Hood K, Bennert K, Channon S, Cohen D, Crowne E, Hambly H, Hawthorne K, Longo M, et al: Development and Evaluation of a Psychosocial Intervention for Children and Teenagers Experiencing Diabetes (DEPICTED): a protocol for a cluster randomised controlled trial of the effectiveness of a communication skills training programme for healthcare professionals working with young people with type 1 diabetes. BMC Health Serv Res 10: 36, 2010.

12. Jahn P, Renz P, Stukenkemper J, Book K, Kuss O, Jordan K, Horn I, Thoke-Colberg A, Schmoll HJ and Landenberger M: Reduction of chemotherapy-induced anorexia, nausea, and emesis through a structured nursing intervention: a cluster-randomized multicenter trial. Support Care Cancer 17: 1543-1552, 2009.

13. Kumakech E, Cantor-Graae E, Maling S and Bajunirwe F: Peer-group support intervention improves the psychosocial well-being of AIDS orphans: cluster randomized trial. Soc Sci Med 68: 1038-1043, 2009. 